\title{
QUALIDADE DE VIDA DE PACIENTES TRANSPLANTADOS RENAIS APÓS LONGO PERÍODO DO TRANSPLANTE
}

\section{Gabrieli Costa Beber}

Fisioterapeuta graduada pela Universidade Regional do Noroeste do Estado do Rio Grande do Sul, Ijuí (RS), Brasil.

\section{Paula Caitano Fontela}

Mestra em Ciências Pneumológicas pela Universidade Federal do Rio Grande do Sul, Porto Alegre (RS), Brasil.

\section{Gerli Elenise Gehrke Herr}

Mestra em Atenção Integral à Saúde pela Universidade Regional do Noroeste do Estado do Rio Grande do Sul; Docente no Departamento de Ciências da Vida na Universidade Regional do Noroeste do Estado do Rio Grande do Sul, Ijuí (RS), Brasil.

\section{Eliane Roseli Winkelmann}

Doutora em Ciências Cardiovasculares pela UFRGS/ RS. Docente do Programa de Pós Graduação Scricto sensu Mestrado em Atenção Integral a Saúde (PPGAIS) - UNICRUZ/UNIJUI, Brasil.

E-mail: elianew@unijui.edu.br
RESUMO: O objetivo do estudo foi avaliar a qualidade de vida (QV) de pacientes pós-transplante renal por meio de protocolo genérico e específico. A avaliação da QV foi realizada por meio de protocolo genérico (Medical Outcomes Study 36-Item Short-Form Health Survey - SF-36) e específico (Kidney Disease Quality of Life Short Form KDQOL-SF). Foram avaliados 24 pacientes. Escores mais baixos foram obtidos nos domínios situação de trabalho $(45,6 \pm 36,6)$, papel físico $(63,5 \pm 42,3)$, dor $(67,0 \pm 26,3)$, composição física $(46,8 \pm 8,7)$ e composição mental $(48,9 \pm 10,8)$ no protocolo KDQOL; e na saúde mental $(53,3 \pm 8,2)$, dor $(63,5 \pm 26,8)$, aspecto físico $(63,5 \pm 42,3)$ e capacidade funcional $(64,4 \pm 22,2)$ no protocolo SF-36. A QV em geral é boa nos pacientes transplantados renais com prejuízo nos domínios referentes às questões físicas, mentais e relacionadas à dor. O protocolo genérico obteve uma pontuação mais baixa que o protocolo específico. Houve correlação entre o questionário de QV específico e o genérico nos domínios correspondentes.

PALAVRAS-CHAVE: Insuficiência renal crônica; Transplante de rim; Qualidade de vida; Avaliação em saúde.

\section{LIFE QUALITY OF KIDNEY TRANSPLANT PATIENTS AFTER A LONG TRANSPLANT PERIOD}

\begin{abstract}
Current analysis assesses life quality (LQ) of kidney posttransplant patients by generic and specific protocol. LQ assessment was undertaken by generic (Medical Outcomes Study 36-Item ShortForm Health Survey - SF-36) and specific (Kidney Disease Quality of Life Short Form - KDQOL-SF) protocols with 24 patients. Lowest scores occurred within the domains work condition (45.6 \pm 36.6$)$, physical role $(63.5 \pm 42.3)$, pain $(67.0 \pm 26.3)$, physical $(46.8 \pm 8.7)$ and mental $(48.9 \pm 10.8)$ composition in KDQOL protocol; mental health $(53.3 \pm 8.2)$, pain $(63.5 \pm 26.8)$, physical aspect $(63.5 \pm 42.3)$ and functional capacity (64.4 22.2$)$ in SF-36 protocol. As a rule, LQ is good in kidney transplanted patients with liabilities in domains related to physical, mental and pair issues. Generic protocol obtained low scores than specific protocol. There was a co-relationship between specific and generic LQ questionnaire in the corresponding domains.
\end{abstract}

KEY WORDS: Renal insufficiency, chronic; Kidney transplantation; Quality of life; Health evaluation. 


\section{INTRODUÇÃO}

Dados internacionais apontam que houve aumento significativo na realização de transplantes renais (Tx) e na lista de espera de pacientes a transplante (AXELROD et al., 2010). Em alguns pacientes, esses procedimentos se configuram como o único recurso para a manutenção da vida. Porém, essa opção de tratamento nem sempre está disponível para aqueles que aguardam um transplante de órgão, já que o transplante requer um doador (MARINHO; CARDOSO; ALMEIDA, 2010). De acordo com o Registro Brasileiro de Transplantes, em dezembro de 2016, havia 21.264 pacientes na lista de espera para a realização de transplante renal. Desses, 5.492 (25,8\%), foram submetidos ao procedimento (ASSOCIAÇÃO BRASILEIRA DE TRANSPLANTES DE ÓRGÃOS).

Com a evolução de programas preventivos e terapêuticos e o aumento da sobrevida dos receptores de órgãos, tem-se buscado mensurar a qualidade de vida relacionada à saúde (QVRS) após a realização dos transplantes (GARCIA; HARDEN; CHAPMAN, 2012). A avaliação da QVRS tem-se tornado um desfecho relevante para auxiliar a identificar e priorizar os problemas do paciente nos aspectos físico, social e psicológico, permitindo adequar intervenções terapêuticas com o objetivo de melhorar o grau de satisfação do paciente com sua saúde e seu tratamento.

Portanto, o objetivo deste estudo foi avaliar a QVRS de pacientes transplantados renais após longo prazo do transplante, por meio de protocolo genérico e específico, a fim de correlacioná-los.

\section{METODOLOGIA}

Estudo transversal, de abordagem quantitativa realizado na Unidade de Nefrologia de um Hospital Porte IV da região Noroeste do Estado do Rio Grande do Sul, durante o período de agosto de 2013 a setembro de 2014. A pesquisa foi projetada de acordo com as Diretrizes e Normas Regulamentadoras de Pesquisas Envolvendo Seres Humanos, segundo a Resolução do Conselho Nacional de Saúde (CNS) nº. 466/11, submetida e aprovada pelo
Comitê de Ética e Pesquisa da Universidade Regional do Noroeste do Estado do Rio Grande do Sul - Unijuí sob parecer consubstanciado $\mathrm{n}^{\mathrm{0}}$ 542.531/2014.

Durante o período do estudo, 55 pacientes transplantados renais estavam em acompanhamento na Unidade de Nefrologia do referido hospital. Foram considerados critérios para inclusão no estudo: estar em acompanhamento médico na unidade, ter decorrido no mínimo um ano após a intervenção cirúrgica, não haver rejeição do rim transplantado e não estar realizando diálise. Os pacientes foram encaminhados pelos médicos nefrologistas da Unidade de Nefrologia do Hospital e os instrumentos foram aplicados após agendamento prévio do paciente na Unidade de Serviço de Saúde denominada Unijuí Saúde, na cidade de Ijuí, RS, Brasil.

Após a assinatura do termo de consentimento livre e esclarecido foram coletadas informações referentes aos dados do perfil sociodemográfico dos pacientes e aplicados os questionários para avaliação da QVRS. Quanto ao perfil, foram coletados os dados pessoais (nome completo, data de nascimento, idade, sexo, raça, ocupação e medicações em uso). As medicações em uso foram verificadas a partir do relato do paciente mediante a prescrição médica de rotina. Também foram questionados quanto à presença de fatores de risco cardiovascular: hipertensão arterial sistêmica (HAS), diabetes mellitus (DM), dislipidemia, etilismo, tabagismo, estresse (avaliado a partir do relato do paciente considerando-se ter/ser ou não) e para todos esses itens foi questionado o tempo em que se encontrava nessa condição, sedentarismo (considerado sedentário o indivíduo que realiza menos que 03 vezes por semana menos que $30 \mathrm{~min}$. de exercício aeróbio), idade acima de 60 anos e história familiar de doença cardiovascular, patologia de base que deu origem à doença renal, tipo de doador (vivo ou cadáver) e tempo de realização do Tx.

A avaliação da QVRS foi realizada por meio de dois protocolos, sendo um protocolo genérico (Medical Outcomes Study 36-Item Short-Form Health Survey - SF36) e o outro específico (Kidney Disease Quality of Life Short Form - KDQOL-SFTM). O protocolo SF-36 envolve diretamente o paciente e a percepção que este tem sobre a saúde e a QV, validado no Brasil por Ciconelli et al. (1999). Trata-se de um questionário multidimensional 
formado por 36 itens e 11 questões, englobados em oito escalas, componentes ou domínios, que inclui: capacidade funcional (CF), aspecto físico (AF), dor física (DF), estado geral de saúde (GS), vitalidade (VT), aspecto social (AS), aspecto emocional (AE) e saúde mental (SM), em que cada componente ou domínio é avaliado separadamente. Apresenta um escore final de 0 a 100, na qual 0 corresponde ao pior estado geral de saúde e 100 o melhor estado de saúde. Foi desenvolvido para serem utilizadas em grupos de indivíduos, independente da faixa etária, patologia, tratamento, raça e sexo.

O protocolo específico para doença renal, Kidney Disease and Quality of Live-Short Form (KDQOL-SFTM) foi traduzido, adaptado e validado para o Brasil por Duarte et al. (2003). É um questionário que inclui as oito escalas do SF-36 com acréscimo de dimensões específicas da DRC, em que é avaliado o funcionamento e o bem-estar dos indivíduos com doença renal crônica terminal e que realizam algum tipo de tratamento dialítico. É composto por 80 itens divididos em 19 escalas, abrangendo vários domínios que são encontrados somente nesse instrumento para estes indivíduos. Em relação ao escore de pontuação da $\mathrm{QV}$, o mais próximo de 0 corresponde ao pior estado de saúde e mais próximo de 100 indica melhor estado de saúde.

Para verificar a representatividade estatística do número de pacientes em estudo adotaram-se como critérios: proporção estimada de 50\%, erro amostral de 5\%, e nível de significância de 5\%. A partir do cálculo do tamanho da amostra, seriam necessários 24 pacientes. Para o processamento dos dados foi utilizado o programa estatístico Statistical Package for Social Science - SPSS (versão 18.0, Chicago, IL, EUA). Na análise estatística todas as variáveis foram testadas quanto à normalidade pelo teste de Shapiro-Wilk. As variáveis quantitativas foram apresentadas por média \pm desvio-padrão e mediana (valor mínimo - valor máximo). Na comparação entre os valores obtidos nos escores dos questionários de QV, utilizou-se o teste Anova medidas repetidas. Para avaliar a correlação entre o questionário de QV genérico e específico utilizouse o teste de correlação de Spermann. Todos os testes foram aplicados com intervalo de confiança (IC) de 95\%. Considerou-se estatisticamente significativo os valores $\operatorname{com} p<0,05$.

\section{RESULTADOS}

Dos 55 pacientes em acompanhamento na Unidade de Nefrologia, 31 pacientes não foram incluídos no estudo pelos seguintes motivos: dois não atendiam aos critérios de inclusão, dois foram a óbito no período do estudo, 11 não aceitaram participar da pesquisa, cinco não compareceram ao serviço para a avaliação e 11 não foram contatados por impossibilidade de contato telefônico para realizar o agendamento. Dos 24 pacientes elegíveis para o estudo, $75 \%$ (18) eram do sexo masculino. A média de idade foi de 51,7 $\pm 10,5$ anos, com idade mínima de 30 e máxima 68 anos. A mediana do tempo de Tx foi de 58,5 meses, com tempo mínimo de 15 e máximo de 251 meses, sendo que a maioria dos transplantados renais foi receptor de doador vivo (75\%). A patologia de base dos pacientes foi a maioria classificada como hipertensiva $(66,7 \%)$, seguida da doença policística com 23,8\% dos transplantados.

Com relação à ocupação dos pacientes, a maioria era aposentada pela patologia (60,9\%). Quanto à presença de fatores de risco cardiovascular, 87,5\% dos pacientes eram hipertensos, 45,9\% diabéticos e $41,7 \%$ dislipidêmicos, sendo que grande parte deles referia administrar medicamentos diuréticos (62,5\%), bloqueadores de canal de sódio (52,2\%), antidiabéticos (43,5\%) e antidislipidêmicos (43,5\%). A maioria dos pacientes transplantados era sedentária (66,7\%), no entanto, nenhum dos pacientes era etilista ou tabagista atualmente. As características sociodemográficas e clínicas dos pacientes estão apresentadas na Tabela 1 .

Tabela 1. Características sócio-demográficas e clínicas dos pacientes transplantados renais

(continua)

\begin{tabular}{lc}
\hline Variáveis & $\begin{array}{c}\text { Pacientes } \\
\text { transplantados }(\mathbf{n}=\mathbf{2 4})\end{array}$ \\
\hline Idade (anos) & $51,7 \pm 10,5$ \\
Sexo (F/M) - n(\%) & $6 / 18(25,0 / 75,0)$ \\
Tempo de transplante (meses) & $92,3 \pm 77,8$ \\
\hline Tipo de doador $-\mathbf{n}(\%)$ & \\
\hline \multicolumn{1}{c}{ Vivo } & $18(75,0)$ \\
\multicolumn{1}{c}{ Cadáver } & $6(25,0)$ \\
\hline
\end{tabular}


(conclusão) prevaleceram os domínios papel físico e dor.

\begin{tabular}{lc}
\hline Fatores de Risco - $\mathbf{n}(\%)$ & $5(20,8)$ \\
\hline Idade > 60 anos & $21(87,5)$ \\
HAS & $192,1 \pm 124,1$ \\
Tempo de diagnóstico HAS & $11(45,9)$ \\
(meses) & $138,2 \pm 202,4$ \\
Diabetes Mellitus - $\mathbf{n}(\%)$ & $10(41,7)$ \\
Tempo de DM (meses) & $0(0,0)$ \\
Dislipidemia - n(\%) & $6(25,0)$ \\
Tabagismo - n(\%) & $0(0,0)$ \\
Ex-tabagista - n(\%) & $0(0,0)$ \\
Etilismo - n(\%) & $8(33,3)$ \\
Ex-etilista - $\mathbf{n}(\%)$ & $16(66,7)$ \\
Estresse - n(\%) & \\
Sedentário - n(\%) & $2(8,3)$ \\
\hline Medicamentos - n(\%) & $15(62,5)$ \\
\hline Ansiolítico & $3(12,5)$ \\
Diuréticos & $4(17,4)$ \\
Antidepressivo & $8(34,8)$ \\
Nitratos Orgânicos & $10(43,5)$ \\
$\boldsymbol{\beta}$ Bloqueador & $5(21,7)$ \\
Anti-dislipidêmicos & $10(43,5)$ \\
Anti-inflamatório & $12(52,2)$ \\
Antidiabético & \\
Bloqueador de canal de \\
sódio
\end{tabular}

F: feminino; M: masculino; $\beta$ : beta; HAS: hipertensão arterial sistêmica.

A qualidade de vida dos pacientes transplantados renais após longo prazo do transplante é boa na maioria dos domínios, com prejuízo acentuado nos domínios referentes às questões físicas, mentais e relacionadas à dor. Observa-se que a pontuação abaixo de 50 pontos ocorreu somente no questionário específico nos domínios situação de trabalho, composição física e mental. A pontuação entre 50 a 70 pontos foram observadas no questionado específico nos domínios papel físico e dor. Esta faixa foi a mais prevalente no questionário geral, incluindo os domínios capacidade funcional, aspecto físico, dor, estado geral de saúde e saúde mental. Portanto, para ambos os questionários, nesta faixa de pontuação,

Ao comparar o desempenho dos questionários para avaliação da QVRS em pacientes transplantados renais, observa-se diferença estatisticamente significativa no aspecto físico entre as médias das pontuações do questionário específico (KDQOL-SF) e genérico (SF-36) $(p<0,05)$ (Tabela 2).

Tabela 2. Pontuação média obtida pelos pacientes transplantados renais nos domínios do questionário de qualidade de vida específico KDQOL-SF e do questionário de qualidade de vida genérico SF-36

\begin{tabular}{|c|c|c|c|}
\hline $\begin{array}{l}\text { Qualidade de Vida } \\
\text { Domínios } \\
\text { KDQOL-SF }\end{array}$ & Média $\pm D P$ & $\begin{array}{l}\text { Qualidade de } \\
\text { Vida Domínios } \\
\text { SF-36 }\end{array}$ & Média $\pm \mathrm{DP}$ \\
\hline $\begin{array}{l}\text { Sintomas e } \\
\text { problemas }\end{array}$ & $88,8 \pm 12,8$ & $\begin{array}{l}\text { Capacidade } \\
\text { funcional }\end{array}$ & $64,4 \pm 22,2$ \\
\hline Efeitos da doença & $90,5 \pm 8,3$ & Aspecto físico & $63,5 \pm 42,3$ \\
\hline $\begin{array}{l}\text { Sobrecarga da } \\
\text { doença }\end{array}$ & $75,8 \pm 26,9$ & Dor & $63,5 \pm 26,8$ \\
\hline $\begin{array}{l}\text { Situação de } \\
\text { trabalho }\end{array}$ & $45,6 \pm 36,6$ & $\begin{array}{l}\text { Estado Geral de } \\
\text { Saúde }\end{array}$ & $69,9 \pm 24,4$ \\
\hline Função cognitiva & $86,4 \pm 14,0$ & Vitalidade & $71,7 \pm 20,2$ \\
\hline Interação social & $81,9 \pm 19,6$ & Aspecto Social & $77,7 \pm 23,8$ \\
\hline Função sexual & $90,6 \pm 15,5$ & $\begin{array}{l}\text { Aspecto } \\
\text { Emocional }\end{array}$ & $70,8 \pm 39,7$ \\
\hline Sono & $77,4 \pm 19,5$ & Saúde Mental & $53,3 \pm 8,2$ \\
\hline Apoio social & $89,8 \pm 14,0$ & & \\
\hline Saúde no geral & $75,2 \pm 16,5$ & & \\
\hline Função física & $78,9 \pm 22,7$ & & \\
\hline Papel físico & $63,5 \pm 42,3$ & & \\
\hline Dor & $67,0 \pm 26,3$ & & \\
\hline $\begin{array}{l}\text { Estado geral de } \\
\text { saúde }\end{array}$ & $72,3 \pm 21,8$ & & \\
\hline $\begin{array}{l}\text { Bem-estar } \\
\text { emocional }\end{array}$ & $71,0 \pm 24,7$ & & \\
\hline Papel emocional & $70,8 \pm 39,7$ & & \\
\hline Função social & $77,6 \pm 23,9$ & & \\
\hline Energia/fadiga & $71,7 \pm 20,2$ & & \\
\hline Composição física & $46,8 \pm 8,7$ & & \\
\hline $\begin{array}{l}\text { Composição } \\
\text { mental }\end{array}$ & $48,9 \pm 10,8$ & & \\
\hline
\end{tabular}

KDQOL-SF: Kidney Disease and Quality of Live-Short Form; SF-36: Medical Outcomes Study 36-Item Short-Form Health Survey. 
Há 20 domínios no questionário específico e destes em 15 a média foi maior ou igual a 70 pontos. Por outro lado, o questionário genérico tem oito domínios e em três a pontuação foi maior ou igual a 70 pontos. $\mathrm{Na}$ análise entre os questionários, sete domínios são correspondentes, sendo a melhor pontuação observada no questionário específico (estado geral de saúde, aspecto físico, dor, aspecto social). $\mathrm{O}$ aspecto emocional obteve a mesma pontuação em ambos os questionários. $\mathrm{O}$ único domínio do questionário específico com pontuação menor que o questionário genérico foi o aspecto mental, mas não significativo estatisticamente (Tabela 3).

Tabela 3. Comparação entre as médias obtidas nos domínios correspondentes do questionário de qualidade de vida genérico e específico em pacientes transplantados renais

\begin{tabular}{|c|c|c|c|}
\hline \multirow[b]{2}{*}{ Domínios } & \multicolumn{3}{|c|}{ Questionário de Qualidade de Vida } \\
\hline & $\begin{array}{c}\text { KDQOL-SF } \\
\text { média } \pm \text { DP } \\
\text { mediana (valor } \\
\text { mín e valor máx) }\end{array}$ & $\begin{array}{c}\text { SF-36 } \\
\text { média } \pm \text { DP } \\
\text { mediana (valor } \\
\text { mín e valor } \\
\text { máx) }\end{array}$ & $p$ \\
\hline \multirow[b]{2}{*}{$\begin{array}{l}\text { Estado geral de } \\
\text { saúde }\end{array}$} & $75,2 \pm 16,5$ & $69,9 \pm 24,4$ & \multirow[b]{2}{*}{0,132} \\
\hline & $80,0(40,0-100)$ & $\begin{array}{c}72,0(15,0- \\
100)\end{array}$ & \\
\hline \multirow{2}{*}{ Aspecto físico } & $78,9 \pm 22,7$ & $63,5 \pm 42,3$ & \multirow{2}{*}{$0,022^{*}$} \\
\hline & $87,5(25,0-100)$ & $75,0(0,0-100)$ & \\
\hline \multirow[b]{2}{*}{ Aspecto dor } & $67,0 \pm 26,3$ & $63,5 \pm 26,8$ & \multirow[b]{2}{*}{0,357} \\
\hline & $67,5(20,0-100)$ & $\begin{array}{c}62,0(21,0- \\
100)\end{array}$ & \\
\hline \multirow[b]{2}{*}{ Aspecto social } & $81,9 \pm 19,6$ & $77,7 \pm 23,8$ & \multirow[b]{2}{*}{0,258} \\
\hline & $86,7(20,0-100)$ & $\begin{array}{c}75,0(25,0- \\
100)\end{array}$ & \\
\hline \multirow{2}{*}{$\begin{array}{l}\text { Aspecto } \\
\text { emocional }\end{array}$} & $70,8 \pm 39,7$ & $70,8 \pm 39,7$ & \multirow{2}{*}{1,0} \\
\hline & $100,0(0-100)$ & $100,0(0-100)$ & \\
\hline \multirow[b]{2}{*}{ Aspecto mental } & $48,9 \pm 10,8$ & $53,3 \pm 8,2$ & \multirow[b]{2}{*}{0,057} \\
\hline & $51,3(28,3-62,8)$ & $\begin{array}{l}56,0(32,0- \\
64,0)\end{array}$ & \\
\hline
\end{tabular}

*Valores apresentados em média \pm desvio padrão e mediana (valor mínimo e máximo); KDQOL-SF: Kidney Disease and Quality of Live-Short Form; SF36: Medical Outcomes Study 36-Item Short-Form Health Survey; Teste ANOVA medidas repetidas. Considerou-se estatisticamente significativo os valores com $\mathrm{p}<0,05$.

$\mathrm{Na}$ correlação entre o questionário de QV específico com os domínios correspondentes do questionário de QV genérico constata-se correlação entre todos os domínios. Ocorreu correlação forte em todos os domínios, exceto entre composição mental e saúde mental que apresentou correlação regular $(r=0,477)$. Entre o papel emocional e o aspecto emocional houve uma correlação plena $(\mathrm{r}=1,0)$ (Tabela 4).

Tabela 4. Correlação entre os domínios do questionário de qualidade de vida específico com os domínios correspondentes do questionário de qualidade de vida genérico em pacientes transplantados renais

\begin{tabular}{lcc}
\hline $\begin{array}{l}\text { Questionário específico de QV (KDQOL-SF) } \\
\text { - Questionário genérico de QV (SF-36) }\end{array}$ & r & $\boldsymbol{p}$ \\
\hline $\begin{array}{l}\text { Estado geral de saúde - Estado geral de } \\
\text { saúde funcional }\end{array}$ & 0,607 & 0,002 \\
Função física - Aspecto físico & 0,771 & 0,000 \\
Papel emocional - Aspecto emocional & 1,0 & 0,001 \\
Função social - Aspecto social & 0,626 & 0,001 \\
Composição mental - Saúde mental & 0,477 & 0,019 \\
Dor - Dor física & 0,990 & 0,000 \\
\hline
\end{tabular}

KDQOL-SF: Kidney Disease and Quality of Live-Short Form; SF-36: Medical Outcomes Study 36-Item Short-Form Health Survey; Teste de correlação de Spermann. Considerou-se estatisticamente significativo os valores com $\mathrm{p}<0,05$.

\section{DISCUSSÃO}

Neste estudo observamos que a QVRS dos pacientes transplantados renais após longo prazo do transplante é boa na maioria dos domínios, com prejuízo acentuado nos domínios referentes às questões físicas, mentais e relacionadas à dor. Na comparação dos protocolos de avaliação da QVRS verificamos que o questionário específico para doença renal possui pontuação mais alta comparada ao questionário genérico, embora tenha havido diferença estatisticamente significativa somente no domínio aspecto físico. Ocorreu correlação entre o questionário de QV específico e o genérico nos domínios correspondentes.

A avaliação da QVRS após o transplante renal é sem dúvida um dos principais métodos de avaliação da eficácia do tratamento no doente renal crônico (SCHRIER et al., 1994). O objetivo principal do transplante é proporcionar a máxima qualidade e tempo de vida ao paciente, minimizando os efeitos da doença e também os custos com os cuidados (SIEGAL; GREENSTEIN, 1999). 
No presente estudo, há comparação entre os protocolos genérico e específico; no protocolo genérico os pacientes apresentaram menor pontuação da QV. Portanto, embora seja um questionário genérico, ele pode auxiliar a detectar repercussões negativas na QVRS de pacientes transplantados renais após longo período do transplante, além de ser um questionário mais sucinto para avaliação da QV comparado ao questionário específico.

O protocolo específico KDQOL-SF foi desenvolvido inicialmente para doentes renais crônicos em terapia dialítica, mas este protocolo tem sido cada vez mais utilizado para a avaliação de pacientes transplantados renais, com o objetivo de compará-los com os pacientes em hemodiálise ou diálise peritoneal (BAKEWELL; HIGGINS; EDMUNDS, 2001). O estudo que objetivou determinar a confiabilidade de aplicação do questionário KDQOL-SF em transplantados renais, concluiu que o instrumento é válido e confiável para mensurar a QVRS do doente renal crônico após o transplante, e ainda que a maioria das escalas/dimensões do questionário pode ser usada para comparar a QV entre transplantados e pacientes em diferentes estágios da doença renal crônica (BAROTFI et al., 2006).

Há aspectos positivos na QVRS do transplante renal em comparação a outras terapias de substituição renal (hemodiálise e diálise peritoneal) (ASSOCIAÇÃO BRASILEIRA DE TRANSPLANTES DE ÓRGÃOS). Evidências apontam melhora expressiva da QV dos pacientes após o transplante (FERREIRA; ANES, 2010; FARIAS; MENDONÇA, 2010), sendo prevalente e relevante a melhoria relacionada com os aspectos físico e psicológico dos pacientes (FERREIRA; ANES, 2010), entretanto, no presente estudo tais aspectos foram os que apresentaram maiores prejuízos na avaliação da QVRS. Um estudo (LIPPE et al., 2014) que objetivou avaliar as alterações na QVRS na transição da diálise para o transplante renal e comparar a QVRS nos pacientes transplantados com a da população em geral demonstrou que a QV nos domínios específicos para doença renal crônica melhorou estatística e clinicamente em cinco anos na transição da diálise para o transplante renal, já as alterações nos domínios genéricos da QVRS não foram suficientemente grandes para serem consideradas mudanças clinicamente importantes. Além disso, os pacientes transplantados renais não obtiveram QVRS semelhante à da população geral. No presente estudo não realizamos tal comparação, no entanto, os escores apresentados em nossa amostra de pacientes com mediana de cinco anos de transplante renal foram semelhantes as do estudo supracitado com diferenças mais acentuadas nos domínios vitalidade $\mathrm{e}$ saúde mental do SF-36.

Em transplantados renais, os domínios relacionados aos aspectos físicos parecem ser os mais comprometidos (COSTA; NOGUEIRA, 2014; BOHLKE et al., 2009). Estudo mostrou que o componente físico foi influenciado pela presença de comorbidades tais como hipertensão e diabetes, doenças crônicas bastante prevalentes na nossa amostra (BOHLKE et al., 2009). Além disso, outro estudo demonstrou que a QVRS em pacientes transplantados renais com mais de 60 anos foi significativamente inferior em alguns aspectos quando comparado com indivíduos saudáveis, podendo ter contribuído para tal resultado o fato de que a percepção subjetiva da QVRS após o transplante pode permanecer igual porque muitas vezes os pacientes convivem com outras doenças crônicas associadas (CORNELLA et al., 2008).

A melhora da QVRS de pacientes submetidos ao transplante renal pode estar relacionada à redução de fatores estressores como, por exemplo, pela interrupção do tratamento dialítico e a interferência deste na vida diária, a facilitação da vida profissional pela possibilidade mais ampla de empregos e melhora do apoio social (SIEGAL; GREENSTEIN, 1999). No entanto, no presente estudo, verificamos que mesmo em uma população de pacientes com longo período de transplante, a maioria deles estava sem vida profissional ativa, apresentando repercussão negativa importante no domínio situação de trabalho no questionário específico de avaliação da QVRS. Muitos receptores de rim saudável, após a realização do transplante, passam à condição de beneficiários da seguridade social em gozo de aposentadoria ou auxíliodoença (SIQUEIRA, 2011), corroborando aos achados do presente estudo. Entretanto, essa pode ser uma forma de dificultar o acesso ao mercado formal de trabalho, uma vez que, por lei, o retorno voluntário à atividade laboral implicará em cancelamento automático do benefício previdenciário, a partir da data de retorno.

Este estudo apresenta limitações potenciais. Em 
primeiro lugar, embora a amostra seja representativa para a população estudada, os dados são provenientes de uma amostra pequena e geograficamente específica de pacientes transplantados renais. Além disso, algumas perguntas do questionário específico (KDQOL-SF) não foram aplicadas em decorrência de que este protocolo apresenta questionamentos voltados especificamente a pacientes que estão realizando algum tipo de tratamento dialítico, no entanto, o mesmo foi aplicado previamente em alguns estudos nesta população de pacientes (BAROTFI et al., 2006; LIPPE et al., 2014).

\section{CONCLUSÃO}

A QVRS dos pacientes transplantados renais após longo prazo do transplante é boa na maioria dos domínios, com prejuízo acentuado nos domínios referentes às questões físicas, mentais e relacionadas à dor. Na comparação dos protocolos, genérico e específico, observou-se que no protocolo genérico, os pacientes apresentaram menor pontuação da QVRS nos domínios correlatos, embora estatisticamente significativo somente para o domínio aspecto físico. Ocorreu correlação entre o questionário de qualidade de vida específico e o genérico nos domínios correspondentes.

Sugere-se a realização de mais estudos voltados à qualidade de vida específica para pacientes transplantados renais, para que possa ser elaborado um protocolo específico para esta população, já que o protocolo KDQOL foi desenvolvido para pacientes em tratamento dialítico e atualmente aplicado para pacientes com doença renal.

\section{REFERÊNCIAS}

ASSOCIAÇÃO BRASILEIRA DE TRANSPLANTES DE ÓRGÃOS - ABTO. Acessado em 27 de março de 2017. Disponível em: http://www.abto.org.br/abtov03/default. aspx?mn $=476 \& \mathrm{c}=0 \& \mathrm{~s}=157$

AXELROD, D. A.; MCCULLOUGH, K. P.; BREWER, E. D.; BECKER, B. N.; SEGEV, D. L.; RAO, P. S. kidney and pancreas transplantation in the united states, 1999-2008: the changing face of living donation. Am J Transplant., v. 10, n. 2, p. 987-1002, 2010.
BAKEWELL, A. B.; HIGGINS, R. M.; EDMUNDS, M. E. Does ethnicity influence perceived quality of life of patients on dialysis and following renal transplant? Nephrol Dial Transplant., v.16, p. 1395-401, 2001.

BAROTFI, S.; MOLNAR, M. Z.; ALMASI, C.; KOVACS, A. Z.; REMPORT, A.; SZEIFERT, L.; SZENTKIRALYI, A.; VAMOS, E.; ZOLLER, R.; EREMENCO, S.; NOVAK, M.; MUCSI, I. Validation of the Kidney Disease Quality of Life Short Form questionnaire-kidney transplant patients. J Psychosom Res., v. 60, n. 5, p. 495-504, 2006.

BOHLKE, M.; MARINI, S. S.; ROCHA, M.; TERHORST, L.; GOMES, R. H.; BARCELLOS, F. C.; IRIGOYEN, M. C.; SESSO, R. Factors associated with health-related quality of life after successful kidney transplantation: a population-based study. Qual Life Res., v. 18, n. 9, p. 185-93, 2009.

CICONELLI, R. M.; FERRAZ, M. B.; SANTOS, W.; MEINÃO, I.; QUARESMA, M. R. Tradução para a língua portuguesa e validação do questionário genérico de avaliação de qualidade de vida SF-36 (Brasil SF-36). Rev Bras Reumatol., v. 39, p. 143-50, 1999.

CORNELLA, C.; BRUSTIA, M.; LAZZARICH, E.; COFANO, F.; CERUSO, A.; BARBÉ, M. C.; FENOGLIO, R.; CELlA, D.; STRATTA, P. Quality of life in renal transplant patients over 60 years of age. Transplant Proc., v. 40, p. 1865-6, 2008.

COSTA, J. M.; NOGUEIRA, L. T. Associação entre trabalho, renda e qualidade de vida de receptores de transplante renal no município de Teresina, PI, Brasil. J Bras Nefrol. v. 36, n. 3, p. 332-8, 2014.

DUARTE, O. S.; MIYAZAKI, M. C. O. S.; CICONELLI, R. M.; SESSO, R. Tradução e adaptação cultural do instrumento de avaliação de qualidade de vida para pacientes renais crônicos (KDQOL-SFTM). Rev Assoc Med Bras., v. 49, p. 375-81, 2003.

FARIAS, G. M.; MENDONÇA, A. E. O. Comparando a qualidade de vida de pacientes em hemodiálise e pós transplante pelo "WHOQOL_BREF". Reme - Rev Min Enferm., v. 13, p. 574-83, 2009. 
FERREIRA, P. L.; ANES, E. J. Medição da qualidade de vida de insuficientes renais crônicos: criação da versão portuguesa do KDQOL-SF. Rev Port Saúde Pública., v. 28, p. 31-9, 2010.

GARCIA, G. G.; HARDEN, P.; CHAPMAN, J. The global role of kidney transplantation. J Bras Nefrol., v. 34, p. 1-7, 2012.

LIPPE, N. V. D.; WALDUM, B.; BREKKEL, F. B.; AMRO, A. A. G.; REISÆTER, A. V.; OS, I. From dialysis to transplantation: a 5-year longitudinal study on self-reported quality of life. BMC Nephrology., v. 15, p. 191, 2014.

MARINHO, A.; CARDOSO, S. S.; ALMEIDA, V. V. Geographic disparities in organ transplantation in Brazil. Cad Saúde Pública., v. 26, n. 4, p. 786-96, 2010.

SCHRIER, R. W.; BURROWS-HUDSON, S.; DIAMOND, L.; LUNDIN, P.; MICHAEL, M.; DONALD, L. Measuring, Managing, and Improving Quality in the End-Stage Renal Disease Treatment Setting: Committee Statement. Am J Kidney Dis., v. 24, p. 383-8, 1994.

SIEGAL, B.; GREENSTEIN, S. Compliance and noncompliance in kidney transplant patients: cues for transplant coordinators. J Transpl Coord., v. 9, p. 104-8, 1999.

SIQUEIRA, J. P. F. H. Benefícios decorrentes de auxíliodoença para empregados rurais, urbanos e domésticos: anotações à legislação básica pertinente. Síntese., v. 263, p. 71-86, 2011.

Recebido em: 10 de setembro de 2016 Aceito em: 30 de março de 2017 